\title{
Priorisierung von Immobilienmärkten mittels ELECTRE: Nutzung eines Outranking-Modells im taktischen Portfoliomanagement
}

\author{
Steffen Metzner • Mareen Sawitzky
}

Eingegangen: 12. Juli 2016 / Angenommen: 15. November 2016 / Online publiziert: 30. November 2016 (C) Der/die Autor(en) 2016. Dieser Artikel ist eine Open-Access-Publikation.

Zusammenfassung In Zeiten volatiler Immobilienmärkte und einer hohen Wettbewerbsintensität sind leistungsfähige Systeme der Analyse und Entscheidungsunterstützung unverzichtbar. Entscheidungen zu Investitionsstrategien und Einzelinvestitionen basieren zumeist auf mehreren entscheidungsrelevanten Kriterien. Unterschiedliche immobilienwirtschaftliche Entscheidungsalternativen können dabei durchaus Kriterienausprägungen aufweisen, die eine bestimmte Alternative nicht als stets besser bzw. stets schlechter ausweisen. Klassische finanzwirtschaftliche Modelle oder verbreitete qualitative Verfahren wie das Scoring können die gegebene Komplexität meist nicht angemessen berücksichtigen. Eine Weiterentwicklung immobilienwirtschaftlicher Entscheidungsmodelle ist durch die Übertragung und Spezifizierung multikriterieller Verfahren der Entscheidungsunterstützung möglich. Speziell die Untergruppe des Outranking beschäftigt sich mit der schrittweisen Strukturierung, Ordnung und Priorisierung von komplexen Auswahlalternativen. Als spezifische immobilienwirtschaftliche Fragestellung dient hier die Auswahl und Priorisierung von Zielmärkten im taktischen Portfoliomanagement eines institutionellen Immobilienportfolios mit internationaler Ausrichtung. Die Formalisierung des Entscheidungsproblems „Priorisierung von Zielmärkten“ erfolgt mit dem ELECTRE-Verfahren.

Schlüsselwörter Immobilieninvestments · Portfoliomanagement ·

Entscheidungsunterstützung · Multikriterielle Analyse · Outranking · ELECTRE ·

Planung · Controlling

\footnotetext{
S. Metzner $(\bowtie)$

Bauhaus-Universität Weimar, Weimar, Deutschland

E-Mail: email@steffen-metzner.de

M. Sawitzky

Nordrheinische Ärzteversorgung, Düsseldorf, Deutschland

E-Mail: mareen.sawitzky@gmx.com
} 
JEL-Kennziffern $\mathrm{L} 85 \cdot \mathrm{R} 33 \cdot \mathrm{O} 21 \cdot \mathrm{G} 32$

\title{
Prioritization of real estate investment markets using ELECTRE: Outranking models in the tactical portfolio management
}

\begin{abstract}
Institutional real estate investors have to consider volatile and competive investment markets. Controlling and portfolio management have to keep up with the management system by providing innovative and efficient tools of analysis and decision support. Normally, decision making in the field of investment strategies and individual investments is based on several criterias. Alternative markets or investment properties may have similar or can also show opposed results among the various criterias. Traditional financial models (e.g. dcf) or basic qualitative models (e.g. scoring) fail because of a higher complexity, especially when multiple criteria are to consider. Enhanced decision models for real estate investments can get developed by transfer and specification of common multi-criteria models for decision support. Among the various solutions of multi-criteria analysis, especially outranking models can provide structuring, ranking and prioritization of alternatives with complex characteristics. The paper presents ELECTRE as method which can formally solve the decision problem "prioritizing of target markets in the real estate sector".
\end{abstract}

Keywords Real estate investments · Portfolio management - Decision support · Multi-criteria analysis · Outranking $\cdot$ ELECTRE $\cdot$ Planning $\cdot$ Controlling

\section{Bedarf an multikriteriellen Modellen zur Entscheidungsunterstützung}

Institutionelle Immobilieninvestoren treffen regelmäßig unternehmens- und portfoliobezogene Entscheidungen, die größere Vermögens- und Ertragspositionen über lange Zeiträume beeinflussen. Während häufige bzw. untergeordnete Entscheidungen im täglichen Bestandsmanagement meist anhand von Vorgaben und anderen Standards erfolgen können, benötigen seltene bzw. wesentliche immobilienwirtschaftliche Entscheidungen - z. B. im Rahmen der Neuinvestition oder der Desinvestition eine spezifische analytische Unterstützung bezogen auf den individuellen Einzelfall. Die notwendige Grundlage der Entscheidungsunterstützung sind geeignete Informationen (Erhebung, Planung), die anschließend im Sinne der Zielsetzung bewertet werden (Verdichtung, Vergleich, Priorisierung).

Ein erweiterter Anspruch an die fachliche Qualität und Leistungsfähigkeit der Entscheidungsmodelle speziell im Immobilienportfoliomanagement entsteht aus der Komplexität von Immobilienmärkten, Immobilienanlageprodukten und Bewirtschaftungsprozessen. Für die Unterstützung solcher Entscheidungssituationen eigenen sich Modelle, die durch die Analyse und Aufbereitung von Daten die jeweiligen Entscheidungsalternativen exakt bewerten und eindeutig priorisieren. Gesucht sind somit Kennzahlen, Rangfolgen oder Klassifizierungen, auf deren Grundlage eine Entscheidung rational und nachvollziehbar getroffen werden kann. Entscheidungsrelevant sind zum einen klassische finanzwirtschaftliche Kennzahlen wie Rentabilität 
(i. d. R. differenziert nach Anfangsrendite, Wertänderungsrendite, Total Return, IRR u. a.) und zum anderen qualitative, oft risikobezogene Kriterien wie Standort- und Markt-Ratings.

Entscheidungen zu Investitionsstrategien und Einzelinvestitionen basieren zumeist auf mehreren entscheidungsrelevanten Kriterien. Unterschiedliche immobilienwirtschaftliche Entscheidungsalternativen können dabei durchaus gegensätzliche Kriterienausprägungen aufweisen. Klassische finanzwirtschaftliche Modelle oder einfache qualitative Verfahren wie das sehr häufig verwendete Scoring können die gegebene Komplexität meist nicht angemessen berücksichtigen (Metzner 2013, S. 372).

Eine Weiterentwicklung immobilienwirtschaftlicher Entscheidungsmodelle ist durch die Übertragung und Spezifizierung multikriterieller Verfahren der Entscheidungsunterstützung möglich. Diese Gruppe repräsentiert neben den monokriteriellen Verfahren (z. B. Renditevergleich, Barwertvergleich) und bikriteriellen Verfahren (z. B. Rendite-Risiko-Paare im Markowitz-Modell) eine Dritte Option zur Modellentwicklung im Bereich der Entscheidungsunterstützung (Metzner 2013, S. 141). Speziell die Untergruppe des Outranking beschäftigt sich mit der stringenten Strukturierung, Ordnung und Priorisierung von komplexen Auswahlalternativen. Letztere sind somit in komplexen Entscheidungssituationen grundsätzlich zu präferieren, im immobilienwirtschaftlichen Umfeld jedoch nahezu unbekannt.

Um die Anwendbarkeit zu überprüfen wird nachfolgend das Outranking für ein Marktauswahlproblem modelliert. Dabei wird das taktische Immobilienportfoliomanagement als Umsetzung der strategischen Portfolioallokation verstanden (Wellner 2003, S. 55). Das Modell geht somit von einer typischen Entscheidungssituation im Management eines internationalen Immobilienfonds aus: Die auf der strategischen Ebene bereits festgelegte Allocation von Standorten, Nutzungsarten und anderen, den jeweiligen Zielmarkt abgrenzenden Merkmalen muss im Akquisitionsbereich umgesetzt werden. Aus taktischen (bspw. Phase im Marktzyklus) und kapazitätsbezogenen (bspw. Aufbau regionaler Kompetenzen) Gründen findet i. d. R. eine temporäre Priorisierung von Zielmärkten statt, ohne dass damit grundsätzliche Investmentkriterien wie Marktqualität, mittel- bis langfristige Bandbreiten in der Länderallokation oder zulässige Nutzungsarten außer Kraft gesetzt werden. Die hier besprochene Priorisierung orientiert sich an den kurz- bis mittelfristigen, für die Akquisitionsperiode angenommen Marktbedingungen. Modelle der Entscheidungsunterstützung werden in der taktischen Koordination von Akquisitionsprozessen benötigt, um die relevanten Daten zu Volkswirtschaft, Rechtssystem, Immobilienmarkt und weiteren Faktoren für bestimmte Alternativen (hier Zielländer) zu bewerten und diese damit zu priorisieren. Die Formalisierung des Entscheidungsproblems „Priorisierung von Zielmärkten“ erfolgt mit dem ELECTRE-Verfahren, womit unterschiedliche Konstellationen stringent zu bewerten sind.

\section{ELECTRE als spezifisches multikriterielles Verfahren}

ELECTRE (franz. Elimination Et Choix Traduisant la Realité/engl. Elimination and Choice Expressing the Reality) gilt als eines der ältesten Outranking-Verfahren (Zim- 
Tab. 1 Beschriebene Einsatzgebiete von ELECTRE (Auswahl). (Quelle: Eigene Darstellung)

\begin{tabular}{lll}
\hline Studie/Aufsatz & Branche/Einsatzgebiet & Modellvariante \\
\hline $\begin{array}{l}\text { Buchanan und Vanderpooten, Ranking Projects for an } \\
\text { Electricity Unit, 2007 }\end{array}$ & Energiewirtschaft & ELECTRE III \\
$\begin{array}{l}\text { Huang und Chen, Using the ELECTRE II Method, 2005 } \\
\text { Roussat et al., A sustainable demolition waste manage- } \\
\text { ment, 2009 }\end{array}$ & $\begin{array}{l}\text { Hafenwirtschaft } \\
\text { Umweltmanagement }\end{array}$ & ELECTRE II \\
$\begin{array}{l}\text { Rogers, Housing construction process, 2000 } \\
\text { Damaskos und Kalfakakou, Bank branch network, 2005 }\end{array}$ & Bankwesen \\
$\begin{array}{l}\text { Giannoulis und Ishizaka, Decision Support System, 2010 } \\
\text { Botti und Peypoch, Destination competitiveness, 2013 }\end{array}$ & Bildungswesen & ELECTRE III \\
$\begin{array}{l}\text { Metzner und Sawitzky, ELECTRE-Modell zur Priorisie- } \\
\text { rung, 2015 }\end{array}$ & Tourismus & ELECETRE III \\
\hline
\end{tabular}

mermann et al. 1991, S. 207; Benayoun et al. 1966). Die Entwicklung des ersten ELECTRE-Verfahrens geht auf Bernard Roy zurück, der Mitte der 60er-Jahre als Berater bei der Société d'Economie et de Mathématiques Appliquées mit dem Lösen von Entscheidungsproblemen, speziell in der Priorisierung neuer unternehmerischer Tätigkeitsfelder, beauftragt war (Figueira et al. 2005, S. 134). Darauf aufbauend wurden für verschiedene Branchen und Einsatzgebiete spezifische ELECTRE-Modelle entwickelt (Tab. 1).

ELECTRE kann originär quantitative sowie quantifizierbare qualitative Kriterien gleichermaßen verarbeiten und damit parallel zum Lösen eines Entscheidungsproblems nutzen. Anders als beim Scoring müssen Kriterien mit unterschiedlichen Messeinheiten (z. B. Euro, Quadratmeter, Kilometer, Prozent oder Punktwerte) nicht vorab auf eine einheitliche Skala transformiert werden (Figueira et al. 2013, S. 68). Insofern entstehen weniger Informationsverluste. Weiterhin wird die im Scoring kritisierte Kompensation von gegenläufigen Kriterienausprägungen vermieden. Faktisch gegebene Ausschlusskriterien bleiben als eine Art Filter wirksam. Durch die erweiterte Abstufung des Präferenzbegriffs (strikte Präferenz und Indifferenz sowie zusätzlich in ELECTRE schwache Präferenz und Unvergleichbarkeit) kann eine Bewertung vorgenommen werden, die sich enger an den Präferenzen des Entscheiders orientiert.

Das Modell ermittelt eine partielle Präordnung (Quasiordnung), in der auch unvergleichbare Alternativen abgebildet werden. Diese Präordnung ist eine reflexive und transitive Relation, die von einer bestimmten Alternative ausgeht. Ein denkbarer, hier

Abb. 1 Partielle Präordnung als mögliches Ergebnis im Outranking. (Quelle: Eigene Darstellung, in Anlehnung an Geldermann 1999, S. 101 bzw. Gäfgen 1966, S. 257)

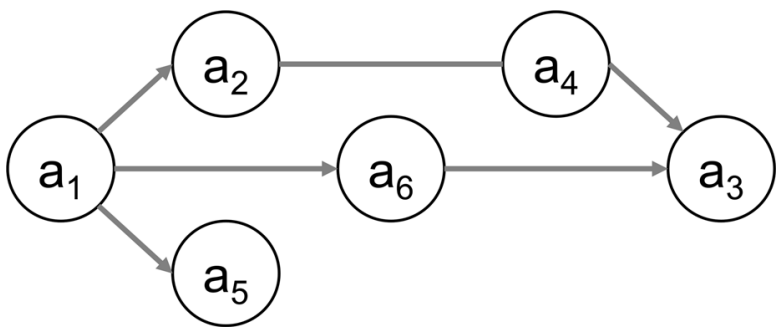


jedoch nicht zwingender Spezialfall ist die vollständige Präordnung (Rangordnung, lineare Anordnung ausgehend von der besten hin zur schlechtesten Alternative) (Roy 1980, S. 490).

Die Darstellung der Präordnung erfolgt durch einen bewerteten Graphen oder durch Tabellendarstellung. Im Beispiel in Abb. 1 dominiert die Alternative $a_{1}$ alle anderen Alternativen. Sind Alternativen indifferent zueinander entfällt die Pfeilspitze wie bei Alternative $\mathrm{a}_{2}$ und $\mathrm{a}_{4}$. Besteht keine Verbindung zwischen Alternativen, so sind diese miteinander unvergleichbar wie es bei Alternative $a_{2}$ und $a_{6}$ der Fall ist (Geldermann 1999, S. 101).

\section{Definition der Bewertungsalternativen und Bewertungskriterien}

Nicht jeder Immobilienmarkt kommt für Investoren in Frage. Während private Investoren zumeist intuitiv entscheiden bzw. faktisch in ihrem Handlungsrahmen auf wenige Regionen bzw. Länder beschränkt sind, nutzen institutionelle Investoren zur ersten Selektion meist vorgelagerte Filter wie Wirtschaftswachstum, Marktgröße und Markttransparenz. Für die verbleibenden Märkte ergibt sich ein Auswahl- bzw. Ordnungsproblem, das sich nicht nur auf eine einzelne Marktkennzahl stützen kann (Nastansky 2012, S. 163 ff). Für die Bestimmung einer Präordnung müssen spezifische Modellkriterien definiert werden (Gäfgen 1966, S. 137 ff). Wichtig ist bei diesem multikriteriellen Modell eine sowohl weitgehende vollständige Abbildung der Entscheidungsgrundlage (Validität) als auch eine Beschränkung auf tatsächlich relevante Kriterien (Stabilität).

\subsection{Bestimmung der Zielmarktalternativen mittels vorgelagertem Filter}

Im Bereich der MADM-Verfahren (Multi-Attributive-Decision-Making) ist die Anzahl der Alternativen im Voraus bekannt (endliche Menge). In dieser Studie werden die potenziellen Zielmärkte eines Immobilienfonds als Alternativen definiert.

Unter einem potenziellen Zielmarkt soll an dieser Stelle ein Land verstanden werden, das für institutionelle Investoren grundsätzlich in Frage kommt. Weltweit gibt es laut UN 193 anerkannte Staaten, die je nach Definition in Industrie-, Transitions- und Entwicklungsländer unterschieden werden können (United Nations 2015). Der IMF definiert zusammenfassend die Kategorien „Advanced Economies“ sowie „Emerging Markets and Developing Countries“ (Nielsen 2011, S. 14 ff). Ein entwickelter Markt weist meist eine höhere Investitionsqualität auf als ein in diesem Sinne rückständiger Markt. Einige branchenbezogene Analysten liefern dazu differenzierte Kennzahlen (z. B. Transparenz-Indizes). Diese können zur Vorauswahl grundsätzlich geeigneter Zielmärkte entsprechend der Investitionsstrategie dienen. Diese vorgelagerten Filter reduzieren die Komplexität des Gesamtmodells. Beispielhaft sollen die nachfolgenden 10 Länder in Tab. 2 rein zur Demonstration des Modells als vorausgewählt gelten. Die Liste beinhaltet hinreichend transparente und entwickelte Länder. Für die interne Ergebnisbeurteilung wurde sie von einem realen, global diversifizierten Portfolio abgeleitet. Je nach Sitz und Statuten anderer Fondsgesellschaften 
Tab. 2 Für das ELECTRE-Modells beispielhaft verwendete Zielmärkte. (Quelle: Eigene Darstellung)

\begin{tabular}{llll}
\hline Europa & Abkürzung & Nordamerika & Abkürzung \\
Deutschland & DE & Kanada & CA \\
Finnland & FI & USA & US \\
Frankreich & FR & - & - \\
Großbritannien & GB & Asien-Ozeanien & Abkürzung \\
Niederlande & NL & Australien & AU \\
Österreich & AT & - & - \\
Schweiz & CH & - & - \\
\hline
\end{tabular}

werden diese Zielmärkte ebenso oder aber nur eingeschränkt in Frage kommen (z. B. rechtliche Beschränkung, steuerliche Konstruktion).

\subsection{Bestimmung der Bewertungskriterien durch empirische Studien}

In der bisherigen immobilienökonomischen Forschung finden sich zahlreiche Studien zu Einflussfaktoren auf den Immobilienmarkt und zu entsprechenden immobilienwirtschaftlichen Entscheidungskriterien. Beispielhaft seien folgende Quellen in Tab. 3 genannt:

Tab. 3 Bisherige Studien zu Investitionskriterien (Auswahl). (Quelle: Eigene Zusammenstellung, Einzelquellen in Anlehnung an Lieser 2012, S. 5)

\begin{tabular}{|c|c|c|}
\hline Themengebiet & $\mathrm{Nr}$ & Studie/Aufsatz \\
\hline \multirow[t]{8}{*}{ Analyse von Märkten } & 01 & Keogh und D'Arcy, Market maturity, 1994 \\
\hline & 02 & D’Arcy und Keogh, Property Market, 1998 \\
\hline & 03 & Keogh und D'Arcy, Property Market Efficiency, 1999 \\
\hline & 04 & Lee, Risk of Investing, 2001 \\
\hline & 05 & Lee, Gauging the Investment Potential, 2005 \\
\hline & 06 & Geurts und Jaffe, Risk und Real Estate, 1996 \\
\hline & 07 & Chen und Hobbs, Real Estate Risk Index, 2003 \\
\hline & 08 & Adair et al., Globalization of Real Estate Markets, 1999 \\
\hline \multirow{11}{*}{$\begin{array}{l}\text { Analyse von } \\
\text { Einflusskriterien }\end{array}$} & 09 & Chin et al., Analysis of Barriers, 2006 \\
\hline & 10 & Lim et al., Real Estate Investment, 2006 \\
\hline & 11 & $\begin{array}{l}\text { Falkenbach, Market selection, 2009; } \\
\text { Falkenbach, Motivation and market selection, } 2010\end{array}$ \\
\hline & 12 & Hoskins et al., Macroeconomic Variables, 2004 \\
\hline & 13 & Worzala und Bernasek, European Economic Integration, 1996 \\
\hline & 14 & Mauro, Corruption and Growth, 1995 \\
\hline & 15 & Knack undKeefer, Economic Performance, 1995 \\
\hline & 16 & Webb, Real Estate Investment Acquisition Rules, 1984 \\
\hline & 17 & Bone-Winkel, Das strategische Management, 1994 \\
\hline & 18 & Lynn, Tectonic Forces of Global Real Estate, 2007 \\
\hline & 19 & Worzala, Overseas Property Investments, 1994 \\
\hline \multirow{3}{*}{$\begin{array}{l}\text { Analyse von } \\
\text { Risikofaktoren }\end{array}$} & 20 & Köpplinger und Wolfrum, Risiken im Auslandsgeschäft, 1986 \\
\hline & 21 & Maier, Risikomanagement, 2007 \\
\hline & 22 & Subroweit, Risikoscoring von Gewerbeimmobilienmärkten, 2009 \\
\hline
\end{tabular}




\begin{tabular}{|c|c|c|c|c|c|c|c|c|c|c|c|c|c|c|c|c|c|c|c|c|c|c|c|c|c|c|c|c|c|}
\hline & N & $x$ & $x$ & & $\times$ & $\times$ & $x$ & & $x$ & $\times$ & & & & & $x$ & $x$ & $x$ & $x$ & $\times$ & $x$ & & $\times$ & $\times$ & & & & $x$ & $x$ & $x$ \\
\hline & $\bar{N}$ & & & & & $x$ & & & & $x$ & & & $x$ & & & $x$ & & & & & $x$ & & & & & & & $x$ & \\
\hline & ని & & & $x$ & & & & & & & & & & $x$ & & $x$ & $x$ & & & & $x$ & & & & & & & & \\
\hline & $\stackrel{9}{\leftarrow}$ & & $x$ & & & & & & & $x$ & & & & & & & & & $\times$ & & & & & $\times$ & $\times$ & & & & \\
\hline & $\stackrel{\infty}{\leftarrow}$ & & $x$ & & & $x$ & & & & & & & & & & & & & & & & $\times$ & $\times$ & & & & & & \\
\hline & $\mp$ & & $x$ & $x$ & & & & & & & & & $x$ & & & & & & & & & $x$ & & & & & $x$ & & \\
\hline & $\div$ & & & & & & & & & & & & & & & & & & $\times$ & & & & & & & & & & \\
\hline & 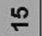 & & & & $x$ & & & & & & & & & & & $\times$ & & & & & & & & & & & & & \\
\hline & $\nabla$ & & & & $x$ & & & & & & & & & & & & & & & $x$ & & & & & & & & & \\
\hline & $\stackrel{m}{=}$ & & & & $\times$ & $x$ & $x$ & & & $\times$ & & & & & $x$ & & & & $\times$ & & & & & & & & & & \\
\hline & $\simeq$ & & & & $\times$ & $\times$ & $x$ & 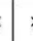 & & $\times$ & & & & & & & & & & & & & & & & & & & \\
\hline & $\mp$ & & $x$ & & $x$ & & & & & & & & & & & $x$ & & & $\times$ & & & & & & & & $x$ & $x$ & $x$ \\
\hline & 우 & & & & & & & & & & & & & $x$ & $x$ & & $x$ & & & & & & & $\times$ & $\times$ & & & & \\
\hline & a & $\times$ & $\times$ & $\times$ & & & & & & $x$ & & & & x & $\times$ & $x$ & $x$ & & $\times$ & $x$ & & & & & $x$ & & & & $x$ \\
\hline & $\infty$ & & $\times$ & $\times$ & $\times$ & & & & & & $x$ & & & & $\times$ & $x$ & & & & & & & & & & & & & \\
\hline & N & $\times$ & $x$ & & $x$ & & & & & & & $\times$ & $x$ & & & & & & & & & & & & & & $x$ & & \\
\hline & 0 & & & & & & & & & & & $x$ & & $\times$ & & $x$ & $\times$ & & & $\times$ & & & & & & & & & \\
\hline & in & & $x$ & & $\times$ & $\times$ & & & & & $x$ & & & $x$ & & $x$ & $x$ & & $x$ & & & & & & & $x$ & $\times$ & & \\
\hline & + & $x$ & $x$ & & & & & & & $x$ & & & & & & $x$ & $x$ & $\times$ & $x$ & & & & & & & & & & \\
\hline 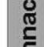 & $m$ & $\times$ & $\times$ & & & & & & & & & & & & & & & & & & & & & & & & & & \\
\hline ثَ & $N$ & $\times$ & $\times$ & & & & & & & & & & & & & & & & & & & & & & & & & & $x$ \\
\hline & - & $x$ & $x$ & & & & & & & & & & & & & & & & & & & & & & & & & & \\
\hline & 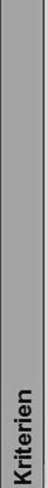 & 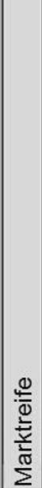 & 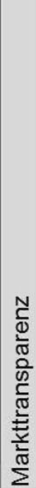 & 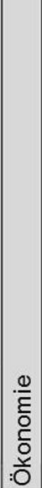 & 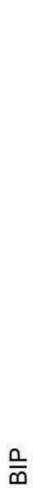 & 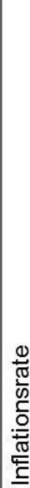 & 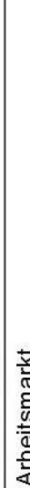 & & $\begin{array}{c}\bar{v} \\
\underline{v}\end{array}$ & 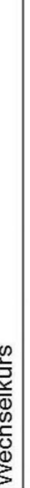 & 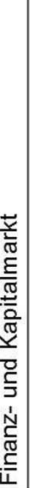 & 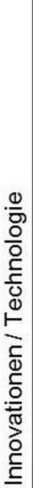 & 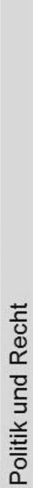 & 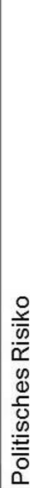 & 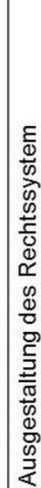 & 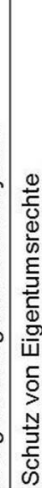 & 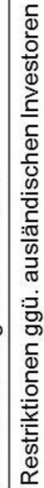 & 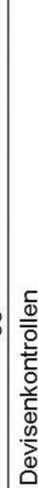 & 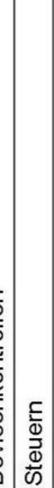 & 동 & 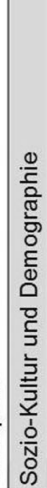 & 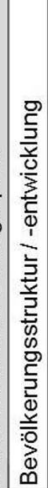 & 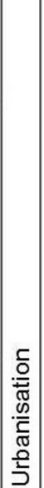 & 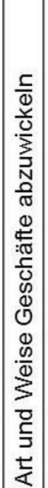 & 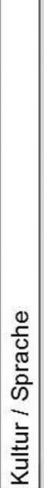 & 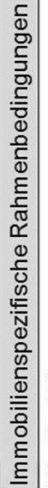 & 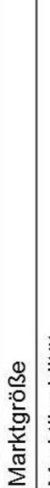 & 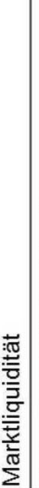 & 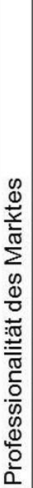 \\
\hline
\end{tabular}

Abb. 2 Aussagen der bisherigen Studien zu Investitionskriterien. (Quelle: Eigene Darstellung)

Häufig führen die Studien zumindest auf einer übergeordneten, stark aggregierten Ebene zu vergleichbaren Ergebnissen. Unterschiede bestehen in den untersuchten Märkten und den statistischen Kennzahlen (z. B. Koeffizienten, Signifikanzen). Auch werden Einflusskriterien bzw. Risiken unterschiedlich differenziert und untergliedert. So nimmt Maier zunächst eine Einordnung nach systematischen und unsystematischen Auslandsrisiken vor und ordnet dann die jeweiligen Risikoarten zu (Maier 
2007, S. 432 ff). Dagegen verwendet Bone-Winkel zuerst die aus dem qualitativen Portfoliomanagement bekannten Kategorien „Marktattraktivität“ und „Wettbewerbsvorteil“" und ordnet dann jeweils Einzelfaktoren zu (Bone-Winkel 1994, S. 182 ff). Andere Systematisierungen orientieren sich an gängigen Modellen der Markt- und Standortanalyse (z. B. Gliederung in Makro- und Mikrostandort) (Urschel 2010, S. 113; Trotz 2004).

Auf der Ebene der Einzelparameter kann es dagegen trotz unterschiedlicher Gliederung wiederum Übereinstimmungen geben. Die nachfolgende Abb. 2 zeigt eine Auswertung der genannten Studien, wobei die Kriterien den Kategorien „Markttransparenz und Marktreife“, „Ökonomie“, „Politik und Recht“, „Sozio-Kultur und Demographie“ sowie ,immobilienspezifische Rahmenbedingungen“ zugeordnet sind. Bei nicht direkt übereinstimmenden (ggf. differenzierteren) Parametern erfolgte hierfür teilweise eine Zusammenfassung bzw. inhaltliche Interpretation.

Die Gesamtschau ermöglicht einen Rückschluss auf potenziell relevante Kriterien. Die folgende Modellierung trifft die Annahme, dass die signifikanten (zur Vereinfachung: die häufigen, plausiblen) Kriterien grundsätzlich eine Unterscheidung von Immobilienmärkten ermöglichen. Eine weitergehende Voruntersuchung war nicht Gegenstand dieser Studie. Vielmehr wird in der Modellierung vorausgesetzt, dass Investoren bestimmte Präferenzstrukturen (Kriterien) definieren und diese dem Portfoliomanagement als zu beachtende Prämisse benennen. Zusätzlich bzw. ersatzweise können auch typische Kriterien zu Marktreife bzw. Marktrisiko einfließen (Keogh und D'Arcy 1994, S. 218).

Generell ist in der praktischen Anwendung darauf zu achten, dass die Anzahl der Kriterien nicht zu hoch ist, ansonsten besteht die Gefahr einer hohen Korrelation, die über entsprechende Mehrfachberücksichtigung von Einflüssen das Ergebnis verzerren kann. Üblicherweise wird im Vorfeld eine Korrelationsanalyse durchgeführt, um Überschneidungen auszuschließen oder die Kriterien entsprechend anzupassen. In der vorliegenden Studie soll auch aus Gründen der Übersichtlichkeit und Nachvollziehbarkeit die Anzahl der Kriterien begrenzt werden. Eine differenziertere Begründung der Auswahl steht hierbei nicht im Mittelpunkt, sollte aber im konkreten Anwendungsfall erfolgen.

\subsection{Verwendung einer einheitlichen Datenbasis}

Für das Entscheidungsmodell müssen die definierten Kriterien mit spezifischen Kennzahlen und Datenquellen unterlegt werden. Die wirtschaftliche Situation kann beispielsweise durch Kennzahlen wie BIP, Inflationsrate oder Arbeitslosenquote beschrieben werden. Schwieriger gestaltet sich die Quantifizierung von Kriterien wie Eigentumsschutz oder Rechtssystem. Bei der Auswahl von Kriterien sind grundsätzlich die Prinzipien der Vollständigkeit, Wesentlichkeit, Überschneidungsfreiheit und Messbarkeit zu beachten (Lifka 2009, S. 50 ff; Bächle 1996, S. 130 ff; speziell zur Modellvereinfachung: Gäfgen 1966, S. 205 ff).

Im internationalen Kontext sind Unterschiede in der Verfügbarkeit und Qualität von Immobilienmarktdaten gegeben (Reichsthaler und Siegel 2009, S. 487). Eine einheitliche Datenbasis kann durch länderübergreifende Datenquellen abgesichert werden. Realistisch ist dies für die meisten volkswirtschaftlichen Parameter, die 
Tab. 4 Im Modell beispielhaft verwendete Kennzahlen und Quellen. (Quelle: Eigene Darstellung, Einzelquellen in Anlehnung an Lieser 2012, S. 86 ff)

\begin{tabular}{|c|c|c|c|c|}
\hline Kriterium & Abk & Quelle & Einheit & Jahr \\
\hline \multicolumn{5}{|l|}{ Ökonomisches Umfeld } \\
\hline BIP pro Kopf & BIPK & IMF & USD & 2014 \\
\hline BIP-Wachstum & BIPW & IMF & $\%$ & $\begin{array}{l}\varnothing 2010- \\
2014\end{array}$ \\
\hline \multicolumn{5}{|c|}{ Immobilienbezogenes Investitionsumfeld } \\
\hline Urbanisationsgrad & URBN & UN & $\begin{array}{l}\% \text { der Ge- } \\
\text { samtbevölke- } \\
\text { rung }\end{array}$ & 2011 \\
\hline $\begin{array}{l}\text { Institutionelles Immobilienver- } \\
\text { mögen pro Kopf }\end{array}$ & IVMK & Pramerica & USD & 2012 \\
\hline \multicolumn{5}{|c|}{ Entwicklungsstand und Tiefe des Kapitalmarktes } \\
\hline $\begin{array}{l}\text { Marktkapitalisierung gelisteter } \\
\text { Unternehmen }\end{array}$ & MKAP & World Bank & $\%$ vom BIP & 2014 \\
\hline $\begin{array}{l}\text { Unabhängigkeit des Finanz- } \\
\text { systems }\end{array}$ & FSYS & $\begin{array}{l}\text { Heritage } \\
\text { Foundation }\end{array}$ & $\begin{array}{l}\text { Score } \\
(0-100)\end{array}$ & 2014 \\
\hline \multicolumn{5}{|c|}{ Schutz des Investors und Qualität des Rechtssystems } \\
\hline $\begin{array}{l}\text { Rechtssicherheit Eigentums- } \\
\text { rechte }\end{array}$ & REGT & $\begin{array}{l}\text { Heritage } \\
\text { Foundation }\end{array}$ & $\begin{array}{l}\text { Score } \\
(0-100)\end{array}$ & 2014 \\
\hline Rechtstaatlichkeitallgemein & RALG & World Bank & $\begin{array}{l}\text { Rating } \\
(-2,5-2,5)\end{array}$ & 2014 \\
\hline \multicolumn{5}{|c|}{ Administrative Hindernisse und rechtliche Restriktionen } \\
\hline Registrierung von Eigentum & RGST & World Bank & $\begin{array}{l}\text { Score } \\
(0-100)\end{array}$ & 2014 \\
\hline Steuerlast & STEU & World Bank & $\begin{array}{l}\text { Score } \\
(0-100)\end{array}$ & 2014 \\
\hline \multicolumn{5}{|c|}{ Sozio-kulturelles und politisches Umfeld } \\
\hline Effektivität der Staatsgewalt & STGW & World Bank & $\begin{array}{l}\text { Rating } \\
(-2,5-2,5)\end{array}$ & 2015 \\
\hline Korruptionsindex & KRPT & $\begin{array}{l}\text { Transparency Internatio- } \\
\text { nal (2013) }\end{array}$ & $\begin{array}{l}\text { Score } \\
(0-100)\end{array}$ & 2014 \\
\hline
\end{tabular}

etwa vom International Monetary Fund (IMF) oder der World Bank bereitgestellt werden. Für die modellbezogenen Bewertungskriterien wurden die nachfolgenden Datenquellen aus Tab. 4 verwendet.

Für die einbezogenen Zielmärkte (Länder) lauten die Daten wie in Tab. 5.

\section{Modellierung des Auswahlproblems mittels ELECTRE III}

Die Verarbeitung der Entscheidungsalternativen, Entscheidungskriterien und Kriterienausprägungen erfolgt in einem strukturierten, in Softwarelösungen programmierbaren Verfahren. Da das ELECTRE-Modell in der immobilienwirtschaftlichen Forschung und Praxis bisher keine Verbreitung aufweist, sollen die Verfahrensschritte und Besonderheiten an dieser Stelle kurz diskutiert werden. Dies erfolgt auszugswei- 
Tab. 5 Daten für die Kriterien und Länder - Gesamtdarstellung. (Quelle: Eigene Darstellung)

\begin{tabular}{lllllllllll}
\hline Krit & AU & DE & FI & FR & GB & CA & NL & AT & CH & US \\
\hline BIPK & 61.063 & 47.716 & 50.038 & 44.288 & 46.313 & 50.253 & 52.225 & 51.433 & 86.146 & 54.360 \\
BIPW & 2,63 & 1,85 & 0,83 & 0,81 & 1,94 & 2,53 & 0,6 & 0,95 & 1,92 & 2,06 \\
URBN & 89,2 & 73,9 & 93,39 & 73,73 & 66,88 & 117,37 & 89,45 & 22,15 & 213,3 & 151,16 \\
IVMK & 30.536 & 19.152 & 20.504 & 18.550 & 21.937 & 23.620 & 20.695 & 20.979 & 35.705 & 23.269 \\
MKAP & 88,59 & 44,94 & 64,2 & 69,8 & 122,7 & 110,7 & 84,5 & 26,9 & 171 & 114,9 \\
FSYS & 90,00 & 70,00 & 80,00 & 70,00 & 80,00 & 80,00 & 80,00 & 70,00 & 80,00 & 70,00 \\
REGT & 90,00 & 90,00 & 90,00 & 80,00 & 90,00 & 90,00 & 90,00 & 90,00 & 90,00 & 80,00 \\
RALG & 1,93 & 1,85 & 2,12 & 1,47 & 1,89 & 1,89 & 1,98 & 1,96 & 2,02 & 1,62 \\
RGST & 77,64 & 72,62 & 85,02 & 60,86 & 73,03 & 75,87 & 76,01 & 83,25 & 87,88 & 82,87 \\
STEU & 78,69 & 73,05 & 85,02 & 69,48 & 87,55 & 90,99 & 83,34 & 72,17 & 86,61 & 75,76 \\
STGW & 1,56 & 1,57 & 1,82 & 1,44 & 1,74 & 1,77 & 1,84 & 1,47 & 2,01 & 1,46 \\
KRPT & 80,00 & 79,00 & 89,00 & 69,00 & 78,00 & 81,00 & 83,00 & 72,00 & 86,00 & 74,00 \\
\hline
\end{tabular}

se und bezogen auf den Anwendungsfall, ansonsten sei auf die allgemeine Literatur zum Verfahren verwiesen (Figueira et al. 2005, S. 133).

\subsection{Auswahl der Variante ELECTRE III}

Grundsätzlich ist hierbei ein Ranking-Problem zu lösen, wobei insgesamt drei Methoden der Entscheidungsunterstützung unterschieden werden können: Selektieren, Sortieren und Ordnen. Je nach Problemstellung des Entscheidungsträgers können bestimmte Varianten eines Entscheidungsmodells bevorzugt werden (Roy 1980, S. $471 \mathrm{f}$ ). Zur Lösung der vorliegenden Ranking-Problematik eignen sich die Varianten ELECTRE II, III und IV. Gemeinsamer Ausgangspunkt für alle ELECTREVarianten sind die festgelegten Bewertungskriterien und Bewertungsalternativen. Variantenspezifische Ausprägungen des Modells ergeben sich aus dem unterschiedlichen Einsatz von Gewichtungskoeffizienten und Schwellen- bzw. Veto-Werten. ELECTRE II verzichtet beispielsweise auf den Einsatz von Schwellenwerten und kommt daher als besonders einfaches Verfahren in Frage. ELECTRE IV ist dagegen geeignet, wenn eine Kriteriengewichtung nicht bestimmt oder darauf verzichtet werden kann (Roy 1991, S. 66 f). ELECTRE III - als Weiterentwicklung von ELECTRE II - berücksichtigt sowohl Schwellen- und Veto-Werte als auch Gewichtungskoeffizienten. Wie bei ELECTRE II werden zwei entgegengesetzte Rangordnungen konstruiert, allerdings rückt das Prinzip der besten Qualifikation in den Mittelpunkt (Roy 1980, S. 495). ELECTRE III wird nachfolgend verwendet, da damit die im Immobilienbereich typische unscharfe bzw. unvollständige Informationslage durch Schwellen- und Veto-Werte gut berücksichtigt werden kann. Weiterhin können die Präferenzen des Entscheidungsträgers durch Gewichtungen gut abgebildet werden (Figueira et al. 2005, S. 139 und 145). ELECTRE III ist ferner übersichtlich und flexibel anpassbar.

Die schrittweise Ableitung einer Präordnung (Abb. 3) der alternativen Zielmärkte beinhaltet nach ELECTRE III zunächst die Abbildung der zentralen Modellparameter (Bewertungsalternativen und -kriterien, Gewichtung sowie Schwellen- und Veto- 


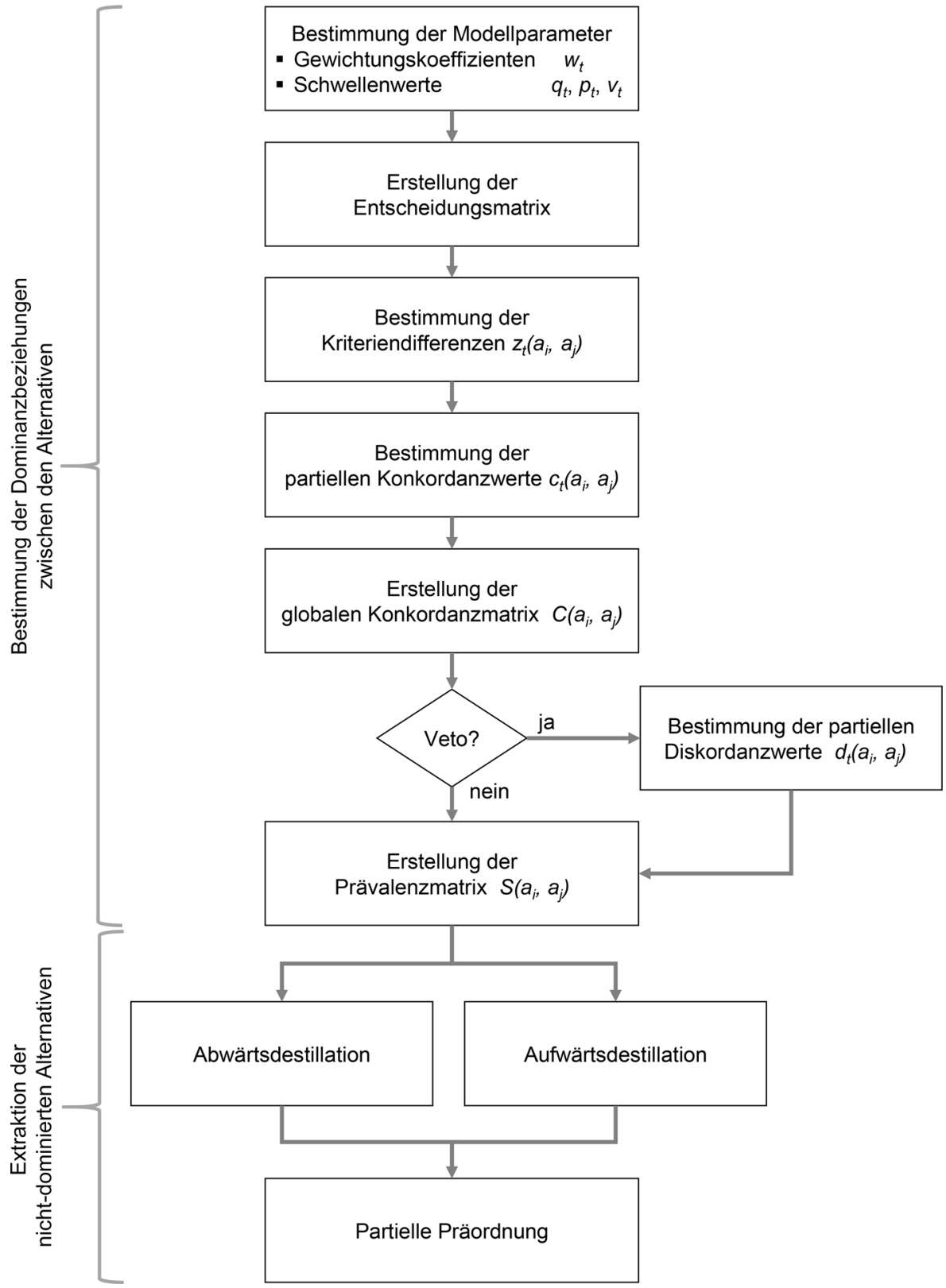

Abb. 3 Verfahrensschritte von ELECTRE III. (Quelle: In Anlehnung an Giannoulis und Ishizaka 2010, S. 490) 
Werte) in einer ersten Matrix (hier bezeichnet als Entscheidungsmatrix). Die jeweiligen Ausprägungen pro Kriterium werden dann verglichen (Differenzmatrizen) und bewertet (partielle Konkordanzwerte). Erst im Anschluss erfolgt eine Gesamtbewertung aller Vor- und Nachteile einer Alternative (Zusammenführung der Kriterien, aggregierte/globale Konkordanzmatrix, Prävalenzmatrix).

Im Einzelnen wird die Vorteilhaftigkeit einer Alternative somit auf Basis der jeweiligen Differenzen pro Kriterium (für ein Alternativenpaar) sowie der kriterienspezifischen Präferenzschwelle ermittelt. Als Gegentests zu diesen Konkordanzen können Vetofunktionen implementiert werden, die eine rechnerisch ermittelte Präferenz aufgrund von Einzelkriterien ggf. ausschließen können. Im Anwendungsfall kann dies z. B. sinnvoll sein, wenn ein Zielmarkt signifikant weniger transparent ist als ein anderer. Eventuelle Vorteile des intransparenten Marktes wie eine geringere Steuerlast können dies dann nicht überkompensieren. Dies führt, anders als bei einfachen qualitativen Modellen (Beispiel Scoring als praxistypisches Modell mit hoher Akzeptanz und Anwendungshäufigkeit (Metzner 2013, S. 372)), zu einer sehr realitätsnahen, an die tatsächlichen Präferenzen orientierte Modellierung. Aus der Kombination der beiden Teilergebnisse Konkordanz (Bestätigung Vorteil) und Diskordanz (Bestätigung Nachtteil) ergibt sich die abschließende Bewertung in der Prävalenzmatrix.

Den Abschluss des ELECTRE-Verfahrens stellt die Rangbestimmung mittels Destillation dar. Diese erfolgt zur Absicherung in zwei Richtungen: Abwärtsdestillation (vom ersten Rang zum letzten Rang) und Aufwärtsdestillation (vom letzten Rang zum ersten Rang). Das Ergebnis bildet die partielle Präordnung.

\subsection{Abbildung der Kriteriendifferenzen}

Die Bildung von Differenzen pro Kriterium und Alternativenpaar bildet den Ausgangspunkt der Bewertung. Die Differenzbildung erfolgt paarweise für jede Alternative und jedes Kriterium $t$ und wird als Matrix $d_{t}\left(a_{i}, a_{j}\right)$ dargestellt, wobei pro Kriterium eine Matrix erstellt wird. Die damit generierte Differenzenmatrix gibt einen ersten Überblick über die Dominanz einzelner Alternativen bezogen auf das jeweils betrachtete Kriterium.

Beispielhaft soll ein Auszug aus der Matrix mit den Differenzen des Kriteriums BIP pro Kopf (BIPK) herangezogen werden. Für das Kriterium BIPK ergibt sich in Tab. 6 folgende Differenzenmatrix.

Die bewertete Alternative repräsentiert die Zeile, der Vergleich findet also in horizontaler Richtung statt. Die Differenzen einer bewerteten Alternative zur jeweils verglichenen Alternative sind spaltenweise abgetragen, d. h. die Differenz zwischen AU (61.062) und DE (47.716) beträgt beim Kriterium BIBK 13.346. Die Differenz zwischen AU (61.062) und FI (50.037) liegt bei 11.025. Die jeweils negierten Werte ergeben sich dann beim Vergleich der Zeile DE zur Spalte AU (-13.346) bzw. der Zeile FI mit AU (-11.025).

Das höchste BIP pro Kopf weist die Schweiz mit 86.145 USD auf. Die in der Zeile $\mathrm{CH}$ abgebildeten Differenzen zu allen anderen Ländern sind dementsprechend durchgängig positiv. Durchgängig negative Differenzen sind bei Frankreich zu sehen, welches mit einem Wert von 44.288 USD das kleinste BIP pro Kopf aufweist. 
Tab. 6 Differenzen Kriterium BIPK. (Quelle: Eigene Darstellung)

\begin{tabular}{|c|c|c|c|c|c|c|c|c|c|c|}
\hline BIPK & $\mathrm{AU}$ & $\mathrm{DE}$ & FI & FR & GB & $\mathrm{CA}$ & $\mathrm{NL}$ & AT & $\mathrm{CH}$ & US \\
\hline $\begin{array}{l}\mathrm{AU} \\
\rightarrow\end{array}$ & 0 & 13.346 & 11.025 & 16.775 & 14.749 & 10.810 & 8838 & 9630 & -25.083 & 6702 \\
\hline $\begin{array}{l}\mathrm{DE} \\
\rightarrow\end{array}$ & -13.346 & 0 & -2321 & 3428 & 1403 & -2537 & -4508 & -3717 & -38.430 & -6644 \\
\hline $\begin{array}{l}\mathrm{FI} \\
\rightarrow\end{array}$ & -11.025 & 2321 & 0 & 5750 & 3724 & -215 & -2187 & -1395 & -36.108 & -4323 \\
\hline $\begin{array}{l}\mathrm{FR} \\
\rightarrow\end{array}$ & -16.775 & -3428 & -5750 & 0 & -2.025 & -5965 & -7937 & -7145 & -41.858 & -10.072 \\
\hline $\begin{array}{c}\mathrm{GB} \\
\rightarrow\end{array}$ & -14.749 & -1403 & -3.724 & 2.025 & 0 & -3.940 & -5.911 & -5.120 & -39.833 & -8047 \\
\hline $\begin{array}{l}\text { CA } \\
\rightarrow\end{array}$ & -10.810 & 2537 & 215 & 5.965 & 3940 & 0 & -1972 & -1.180 & -35.893 & -4108 \\
\hline $\begin{array}{l}\mathrm{NL} \\
\rightarrow\end{array}$ & -8838 & 4508 & 2187 & 7.937 & 5911 & 1972 & 0 & 792 & -33.921 & -2136 \\
\hline $\begin{array}{l}\text { AT } \\
\rightarrow\end{array}$ & -9630 & 3717 & 1395 & 7145 & 5120 & 1180 & -792 & 0 & -34.713 & -2927 \\
\hline $\begin{array}{l}\mathrm{CH} \\
\rightarrow\end{array}$ & 25.083 & 38.430 & 36.108 & 41.858 & 39.833 & 35.893 & 33.921 & 34.713 & 0 & 31.785 \\
\hline $\begin{array}{l}\text { US } \\
\rightarrow\end{array}$ & -6.702 & 6644 & 4323 & 10.072 & 8047 & 4108 & 2136 & 2927 & -31.785 & 0 \\
\hline
\end{tabular}

Deutschland befindet sich im unteren Drittel der verglichenen Länder, nur zwei Differenzen sind positiv. Die Werte in der Diagonalen der Matrix haben keine Relevanz für die Bewertung und sind stets Null. Diese Werte stellen jeweils die Bewertung der entsprechenden Alternative mit sich selbst dar.

\subsection{Abbildung der Präferenzschwellenwerte}

Ein wesentlicher Parameter im Alternativenvergleich pro Kriterium ist die jeweilige Präferenzfunktion. Dabei ist die Indifferenzschwelle $q_{\mathrm{t}}$ diejenige Differenz zwischen den Werten des Kriteriums $\mathrm{k}_{\mathrm{t}}$ zweier Alternativen, bei der aus Sicht des Entscheidungsträgers gerade noch Indifferenz zwischen beiden Alternativen herrscht (Roy et al. 1986, S. 320). Beispielsweise weist die Alternative „Immobilienmarkt DE“ beim Kriterium „Rechtstaatlichkeit“ einen Punktewert von 1,85 auf, die Alternative „Immobilienmarkt GB“ dagegen einen Punktewert von 1,89. Rechnerisch ergibt sich ein Unterschied von 0,04 Punkten. Läge die Indifferenzschwelle bei 0,07 Punkten, würde der unterschiedliche Transparenzgrad unwesentlich sein, d. h. beide Alternativen als gleich gut beurteilt werden. Die Entscheidung zu beiden Alternativen wäre indifferent.

Den zweiten wesentlichen Punkt der Präferenzfunktion bildet die Präferenzschwelle. Die Präferenzschwelle $p_{\mathrm{t}}$ ist diejenige Differenz zwischen den Kriterienwerten zweier Alternativen, ab der eine Alternative gegenüber der anderen präferiert wird (Roy et al. 1986, S. 321; Ishizaka und Nemery 2013, S. 185). Angenommen im obigen Beispiel sollen nun DE (1,85 Punkte) und FR (1,47 Punkte) miteinander verglichen werden. Die Differenz der beiden Alternativen beträgt 0,42 Punkte. Der 
Abb. 4 Beispiel für Präferenz-Schwellenwerte. (Quelle: Eigene Darstellung)

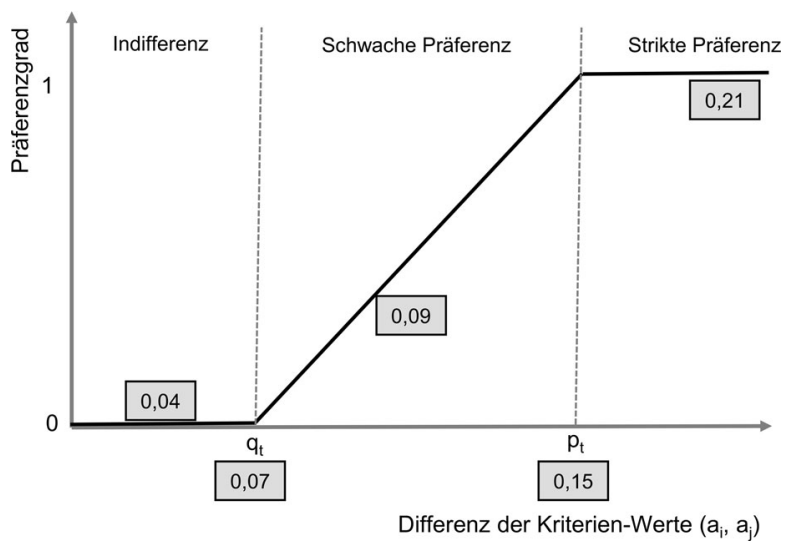

Entscheidungsträger präferiert diejenigen Alternativen, deren Differenz größer oder gleich 0,15 ist. Zunächst kann festgestellt werden, dass die beiden Alternativen nicht indifferent sind, weil die Differenz den Wert von $q_{\mathrm{t}}=0,07$ übersteigt. Gleichzeitig ist die Differenz größer als die Präferenzschwelle $p_{\mathrm{t}}=0,15$. Im Beispiel wäre daher DE gegenüber FR zu bevorzugen, weil es beim Vergleichskriterium als deutlich sicherer gilt.

Liegen Differenzenwerte zwischen den beiden Schwellenwerten $q_{\mathrm{t}}$ und $p_{\mathrm{t}}$, wird dies als schwache Präferenz gewertet. Im obigen Beispiel wäre dies der Fall, wenn die Differenz zweier Alternativen etwa 0,09 Punkte beträgt $(0,07 \leq 0,09 \leq 0,15)$. Der jeweilige Präferenzgrad kann beispielsweise wie in Abb. 4 dargestellt linear zwischen den Punkten 0 und 1 interpoliert werden, andere Funktionsverläufe sind aber denkbar.

Für jedes Kriterium sind die Präferenzschwellen so festzulegen, dass sie die Präferenzen des Entscheidungsträgers widerspiegeln. Allgemein können direkte und indirekte Verfahren zur Bestimmung der Schwellenwerte unterschieden werden. Bei den direkten Verfahren werden die Präferenzen und Einschätzungen des Entscheidungsträgers mit einbezogen. Nachteilig ist hierbei die fehlende Nachvollziehbarkeit für Dritte. Daher bietet sich die Verwendung von indirekten Verfahren an, die nicht direkt auf die Beurteilung des Entscheidungsträgers zurückgreifen. Beispielsweise können statistische Tests bzw. entsprechende Software zur automatisierten Berechnung eingesetzt werden (Figueira et al. 2005, S. 139 und 150 ff). Dennoch ist sowohl die Auswahl der Schwellenwertfunktion als auch die Festlegung der Schwellenwerte häufig subjektiv geprägt und damit ein kritischer Faktor. Der Einfluss auf die finale Präordnung sollte mittels Variante- bzw. Stabilitätstests (z. B. Sensitivitätsanalyse) geprüft werden (Roy et al. 1986, S. 322).

Für alle Kriterien sind die Schwellenwerte gesondert zu bestimmen. Dabei ist es auch zulässig, auf ein Veto zu verzichten. Bei einer einheitlichen softwaretechnischen Implementierung können die Vetoschwellen ersatzweise so gewählt werden, dass die zu erwartenden Differenzen diese nicht erreichen. Die festgelegten Schwellenwerte sind in der nachfolgenden Tab. 7 ersichtlich. 
Tab. 7 Festlegung der Präferenz-Schwellenwerte. (Quelle Eigene Darstellung)

\begin{tabular}{|c|c|c|c|}
\hline Parameter & $\begin{array}{l}\text { Indifferenzschwelle } \\
\left(\mathrm{q}_{\mathrm{t}}\right)\end{array}$ & $\begin{array}{l}\text { Präferenzschwelle } \\
\left(\mathrm{p}_{\mathrm{t}}\right)\end{array}$ & $\begin{array}{l}\text { Bandbreite Länder } \\
(\max -\min )\end{array}$ \\
\hline \multicolumn{4}{|l|}{ Ökonomisches Umfeld } \\
\hline BIP pro Kopf & 3000,00 & 6000,00 & $40.345,00$ \\
\hline BIP-Wachstum & 0,20 & 0,40 & 3,00 \\
\hline \multicolumn{4}{|c|}{ Immobilienbezogenes Investitionsumfeld } \\
\hline Urbanisationsgrad & 5,00 & 10,00 & 22,00 \\
\hline $\begin{array}{l}\text { Institutionelles Immobilienver- } \\
\text { mögen pro Kopf }\end{array}$ & 2500,00 & 5000,00 & $17.155,00$ \\
\hline \multicolumn{4}{|c|}{ Entwicklungsstand \& Tiefe des Kapitalmarktes } \\
\hline $\begin{array}{l}\text { Marktkapitalisierung gelisteter } \\
\text { Unternehmen }\end{array}$ & 15,00 & 30,00 & 144,00 \\
\hline $\begin{array}{l}\text { Unabhängigkeit des Finanzsys- } \\
\text { tems }\end{array}$ & 5,00 & 15,00 & 20,00 \\
\hline \multicolumn{4}{|c|}{ Schutz des Investors \& Qualität des Rechtssystems } \\
\hline Eigentumsrechte & 5,00 & 15,00 & 10,00 \\
\hline Rechtstaatlichkeit & 0,07 & 0,15 & 1,00 \\
\hline \multicolumn{4}{|c|}{ Administrative Hindernisse \& rechtliche Restriktionen } \\
\hline Registrierung von Eigentum & 6,00 & 10,00 & 27,00 \\
\hline Steuerlast & 5,00 & 10,00 & 22,00 \\
\hline \multicolumn{4}{|c|}{ Sozio-kulturelles \& politisches Umfeld } \\
\hline Effektivität der Staatsgewalt & 0,20 & 0,40 & 1,00 \\
\hline Korruptionsindex & 5,00 & 10,00 & 20,00 \\
\hline
\end{tabular}

\subsection{Abbildung der Vetoschwellenwerte}

Die Vetoschwelle $v_{\mathrm{t}}$ kommt im ELECTRE-Modell zum Einsatz, wenn die Kompensation sehr schlechter Kriterien ausgeschlossen werden soll. Die Vetofunktion greift somit ein, wenn die Differenz zwischen den Kriterienwerten zweier Alternativen größer oder gleich der definierten Vetoschwelle ist. Trifft diese Bedingung bei mindestens einem Kriterium zu, wird die Alternative aus dem paarweisen Vergleich ausgesondert.

Angenommen hinsichtlich des Kriteriums BIBK (BIP pro Kopf) werden FR (44.288 USD) und AU (61.062 USD) miteinander verglichen. Der Entscheidungsträger setzt die Vetoschwelle bei 15.000 USD. Die Differenz der beiden Alternativen ist 16.775 USD und überschreitet damit die Vetoschwelle. Demnach ist AU bei diesem Vergleich viel besser als FR. In diesem Fall erhält FR bei diesem Kriterium ein Veto. Wenig stärkere oder wenig schwächere Länder erzeugen dagegen kein Veto. Zwischenwerte sind denkbar, beispielsweise im Fall eine Differenz von 10.000 USD (Abb. 5).

Analog zu den zuvor genannten Überlegungen hinsichtlich der Präferenzschwellen $q_{t}$ und $p_{t}$ spielen auch hier fachliche und subjektive Überlegungen eine Rolle. Beim besprochenen Kriterium „Korruptionsindex“ kommen beispielsweise Erfahrungen und Risikomanagementfragen zum Tragen. Ein Veto könnte demnach schon bei einer Differenz von 20 Punkten eingelegt werden $\left(v_{t}=20\right)$. Bei Erweiterung der 
Abb. 5 Beispiel für Vetoschwellenwerte. (Quelle: Eigene Darstellung)

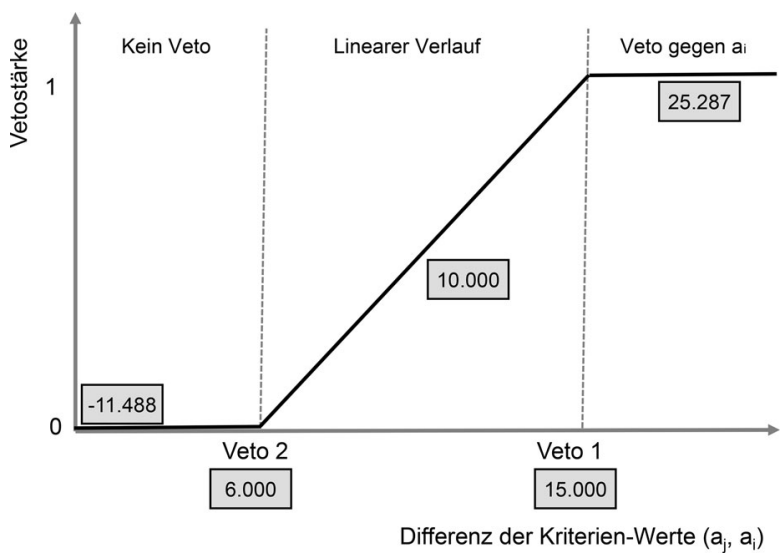

Alternativenbetrachtung auch auf Entwicklungs- bzw. Schwellenländer wäre dies aber zu einengend, so dass in diesem Fall ggf. eine andere Vetoschwelle zu überlegen ist (z. B. $\left.v_{\mathrm{t}}=25\right)$.

Für die Schwellenwerte gilt allgemein $v_{t} \geq p_{t} \geq q_{t} \geq 0$. Die Vetoschwelle muss also größer oder zumindest gleich der Präferenzschwelle sein. Insofern stellt die jeweils umgekehrte Präferenzanalyse ( $a_{i} v s a_{j}$ bzw. $a_{j} v s a_{i}$ ) ein Teilelement der VetoFunktion dar. Aus Gründen der Übersicht wurde diese über eine zweite (untere) Vetoschwelle $u_{t}$ abgebildet (Tab. 8).

\subsection{Ableitung der globalen Konkordanzmatrix}

Kriteriendifferenzen, Präferenzfunktionen und Vetofunktionen führen zu partiellen Präferenzen für alle Kriterien und Paare von Alternativen (Verfahrensschritt aus Platzgründen hier nicht differenziert dargestellt). Diese partiellen (pro Kriterium gebildeten) Konkordanzmatrizen werden im Folgeschritt zu einer globalen Konkordanzmatrix $C\left(a_{\mathrm{i}}, a_{\mathrm{j}}\right)$ aggregiert. Die Aggregation der partiellen Konkordanzwerte erfordert zusätzliche Parameter in Form von relativen Anteilen (Gewichtungen). Zur Bestimmung der Gewichte kommen verschiedene Methoden in Frage, beispielsweise absolute Gewichtung, rangfolgenbasierte Gewichtung oder Point Allocation. Als Gewichte sollen hier die nachfolgenden Werte aus Tab. 9 gelten:

In der Aggregation werden die partiellen Konkordanzwerte der jeweiligen Kriterien $\mathrm{k}_{\mathrm{t}}$ mit dem kriterienspezifischen Gewichtungsfaktor $w_{\mathrm{t}}$ multipliziert (Roy 1980, S. 477). Die globale Konkordanzmatrix ist demnach die gewichtete Summe aller partiellen Konkordanzwerte und misst die Übereinstimmung mit der Hypothese „Alternative $a_{\mathrm{i}}$ ist genauso gut wie oder besser als Alternative $a_{\mathrm{j}}{ }^{\text {" }}$ in Bezug auf alle Kriterien (Ishizaka u.a. 2013, S. 185). Als aggregierte Konkordanzmatrix wie in Tab. 10 dargestellt ergibt sich:

Die Werte in der aggregierten Konkordanzmatrix geben an, inwieweit die entsprechende Zeile die entsprechende Spalte dominiert. Ein Wert von 1,000 bedeutet, dass diejenige Alternative bei allen Kriterien mindestens genauso gut wie oder auch besser als die andere Alternative ist (Metzner 2013, S. 365). Wie in Tab. 10 zu sehen, 
Tab. 8 Festlegung der Vetoschwellenwerte. (Quelle: Eigene Darstellung)

\begin{tabular}{lcc}
\hline Parameter & Vetoschwelle $1\left(\mathrm{v}_{\mathrm{t}}\right)$ & Vetoschwelle $2\left(\mathrm{u}_{\mathrm{t}}\right)$ \\
\hline Ökonomisches Umfeld & $15.000,00$ & 6000,00 \\
BIP pro Kopf & 2,50 & 0,40 \\
BIP-Wachstum & & \\
Immobilienbezogenes Investitionsumfeld & 25,00 & 10,00 \\
Urbanisationsgrad & $15.000,00$ & 5000,00 \\
Institutionelles Immobilienvermögen pro Kopf & \\
Entwicklungsstand \& Tiefe des Kapitalmarktes & & 30,00 \\
Marktkapitalisierung gelisteter Unternehmen & 100,00 & 15,00 \\
Unabhängigkeit des Finanzsystems & 25,00 & \\
Schutz des Investors \& Qualität des Rechtssystems & 15,00 \\
Eigentumsrechte & 20,00 & 0,15 \\
Rechtstaatlichkeit & 0,50 & \\
Administrative Hindernisse \& rechtliche Restriktionen & 10,00 \\
Registrierung von Eigentum & 25,00 & 10,00 \\
Steuerlast & 20,00 & \\
Sozio-kulturelles \& politisches Umfeld & & 0,40 \\
Effektivität der Staatsgewalt & 0,60 & 10,00 \\
Korruptionsindex & 20,00 & \\
\hline
\end{tabular}

ist dies z. B. bei den Paaren (AU, DE), (AU, FR), (AU, AT), (FI, FR), (FI, AT), (CA, DE) usw. der Fall. Bei kleineren Werten kann darauf geschlossen werden, dass die entsprechende Alternative schlechter ist als die andere, wie bei dem Paar (FR, AU) $\mathrm{zu}$ sehen ist. Hierbei bedeutet $(\mathrm{FR}, \mathrm{AU})=0,210$, dass FR weder besser als, noch genauso gut wie AU ist. Dies lässt darauf schließen, dass FR schlechter beurteilt wird als AU.

Eine Interpretation der Konkordanzwerte ist schwierig. Nur im Falle einer Gleichgewichtung aller Kriterien und ohne eindeutige partielle Konkordanzen (nur 0 oder

Tab. 9 Festlegung der Gewichtungen. (Quelle: Eigene Darstellung)

\begin{tabular}{ll}
\hline Kriterium t & Gewichtung $w_{\mathrm{t}}$ \\
\hline BIP pro Kopf & 8,557 \\
BIP-Wachstum & 4,807 \\
Urbanisationsgrad & 6,375 \\
Institutionelles Immobilienvermögen pro Kopf & 6,518 \\
Marktkapitalisierung gelisteter Unternehmen & 9,795 \\
Unabhängigkeit des Finanzsystems & 11,715 \\
Eigentumsrechte & 19,366 \\
Rechtstaatlichkeit & 4,786 \\
Registrierung von Eigentum & 3,352 \\
Steuerlast & 7,370 \\
Effektivität der Staatsgewalt & 2,804 \\
Korruptionsindex & 14,557 \\
\hline
\end{tabular}


Tab. 10 Aggregierte globale Konkordanzmatrix. (Quelle: Eigene Darstellung)

\begin{tabular}{lllllllllll}
\hline AGG & AU & DE & FI & FR & GB & CA & NL & AT & CH & US \\
\hline AU & 1,000 & 1,000 & 0,796 & 1,000 & 0,845 & 0,815 & 0,989 & 1,000 & 0,542 & 0,862 \\
DE & 0,507 & 1,000 & 0,543 & 0,936 & 0,762 & 0,607 & 0,618 & 0,922 & 0,388 & 0,675 \\
FI & 0,681 & 0,952 & 1,000 & 1,000 & 0,854 & 0,760 & 0,965 & 1,000 & 0,639 & 0,746 \\
FR & 0,210 & 0,616 & 0,377 & 1,000 & 0,390 & 0,166 & 0,367 & 0,736 & 0,155 & 0,547 \\
GB & 0,679 & 0,974 & 0,689 & 0,976 & 1,000 & 0,861 & 0,841 & 0,906 & 0,521 & 0,819 \\
CA & 0,791 & 1,000 & 0,838 & 1,000 & 1,000 & 1,000 & 0,988 & 0,988 & 0,613 & 0,896 \\
NL & 0,743 & 0,952 & 0,897 & 0,998 & 0,854 & 0,765 & 1,000 & 0,954 & 0,606 & 0,781 \\
AT & 0,413 & 0,810 & 0,492 & 0,838 & 0,619 & 0,524 & 0,537 & 1,000 & 0,334 & 0,790 \\
CH & 0,893 & 1,000 & 0,982 & 1,000 & 1,000 & 0,952 & 1,000 & 1,000 & 1,000 & 1,000 \\
US & 0,510 & 0,855 & 0,566 & 1,000 & 0,712 & 0,601 & 0,617 & 0,855 & 0,237 & 1,000 \\
\hline
\end{tabular}

1), könnte der Konkordanzwert interpretiert werden als der Prozentsatz von Kriterien, bei der eine Alternative mindestens genauso gut ist wie die andere. Für einen Wert von 0,8333 hieße dies, dass für rund $83 \%$ aller Kriterien (hier 10 von 12) die Alternative $a_{i}$ mindestens genauso gut ist wie die Alternative $a_{j}$.

\subsection{Ableitung des finalen Rankings aus den Präordnungen}

Abschließend kann die finale partielle Präordnung abgleitet werden. Sie ist definiert als der Schnittpunkt aus den beiden Präordnungen (Abb. 6), die zuvor durch die Ab- und Aufwärtsdestillation (aus Platzgründen nicht dargestellt) generiert wurden (Giannoulis und Ishizaka 2010, S. 492).

Der Vergleich der beiden Präordnungen zeigt, dass $\mathrm{CH}$ in jedem Fall Rang 1 in der finalen Rangordnung einnehmen und die letzten fünf Ränge auch einheitlich bestimmt sind. Bei den übrigen Rängen ist nochmals zu prüfen, welche finale Ordnung erzielt wird. Hierfür wird eine Rankingmatrix aufgestellt, in der die Alternativen wieder paarweise miteinander verglichen werden. In der Rankingmatrix wird nun aufgezeigt, ob eine Alternative gegenüber der anderen zu bevorzugen ist

\begin{tabular}{|l|l|l|l|}
\hline \multicolumn{2}{|l|}{ 1. Präordnung: Abwärtsdestillation } & \multicolumn{2}{l|}{ 2. Präordnung: Aufwärtsdestillation } \\
\hline Rang & Alternativen & Rang & Alternativen \\
\hline 1 & Schweiz (CH) & 1 & Schweiz (CH) \\
\hline 2 & Kanada (CA) & 2 & USA (US) \\
\hline 3 & Australien (AU) / Finnland (FI) & 3 & Kanada (CA) \\
\hline 4 & USA (US) & 4 & Australien (AU) / Finnland (FI) \\
\hline 5 & Niederlande (NL) / & 5 & Niederlande (NL) \\
\hline 6 & Großbritannien (GB) & 6 & Großbritannien (GB) \\
\hline 7 & Deutschland (DE) & 8 & Frankreich (FR) \\
\hline 8 & Frankreich (FR) & 9 & Österreich (AT) \\
\hline 9 & Österreich (AT) & & \\
\hline
\end{tabular}

Abb. 6 Vergleich der ersten und der zweiten Präordnung. (Quelle: Eigene Darstellung) 
Tab. 11 Bewertungsschema zur Erstellung der Rankingmatrix. (Quelle: Eigene Darstellung, in Anlehnung an Ishizaka und Nemery 2013, S. 200)

\begin{tabular}{|c|c|c|}
\hline Präferenzsituation & Notation & Definition \\
\hline$a_{\mathrm{i}}$ ist besser als $a_{\mathrm{j}}$ & $a_{\mathrm{i}} \boldsymbol{P}^{+} a_{\mathrm{j}}$ & $\begin{array}{l}a_{\mathrm{i}} \text { hat einen höheren Rang als } a_{\mathrm{j}} \text { in beidenPräordnungen } \\
a_{\mathrm{i}} \text { hat einen höheren Rang als } a_{\mathrm{j}} \text { in einer Präordnung und } \\
\text { ist indifferent } \mathrm{zu} a_{\mathrm{j}} \text { in der anderen Präordnung }\end{array}$ \\
\hline$a_{\mathrm{i}}$ ist indifferent $\mathrm{zu} a_{\mathrm{j}}$ & $a_{\mathrm{i}} \mathbf{I} a_{\mathrm{j}}$ & $a_{\mathrm{i}}$ und $a j$ sind indifferent in beiden Präordnungen \\
\hline $\begin{array}{l}a_{\mathrm{i}} \text { und } a_{\mathrm{j}} \text { sind unver- } \\
\text { gleichbar }\end{array}$ & $a_{\mathrm{i}} R a_{\mathrm{j}}$ & $\begin{array}{l}a_{\mathrm{i}} \text { hat einen höheren Rang als } a_{\mathrm{j}} \text { in einer Präordnung, aber } \\
a_{\mathrm{j}} \text { hat einen höheren Rang als } a_{\mathrm{i}} \text { in der anderen Präordnung }\end{array}$ \\
\hline$a_{\mathrm{i}}$ ist schlechter als $a_{\mathrm{j}}$ & $a_{\mathrm{i}} \boldsymbol{P}^{-} a_{\mathrm{j}}$ & $\begin{array}{l}a_{\mathrm{i}} \text { hat einen niedrigeren Rang als } a_{\mathrm{j}} \text { in beiden Präordnun- } \\
\text { gen } \\
a_{\mathrm{i}} \text { ist indifferent } \mathrm{zu} \mathrm{a}_{\mathrm{j}} \text { in einer Präordnung, aber } a_{\mathrm{j}} \text { hat einen } \\
\text { höheren Rang als } a_{\mathrm{i}} \text { in der anderen Präordnung }\end{array}$ \\
\hline
\end{tabular}

Tab. 12 Rankingmatrix auf Basis der Präferenzsituation. (Quelle: Eigene Darstellung)

\begin{tabular}{lllllllllllll}
\hline Präf & $\mathrm{AU}$ & $\mathrm{DE}$ & $\mathrm{FI}$ & $\mathrm{FR}$ & $\mathrm{GB}$ & $\mathrm{CA}$ & $\mathrm{NL}$ & $\mathrm{AT}$ & $\mathrm{CH}$ & $\mathrm{US}$ & Anz. $P^{+}$ & Rang \\
\hline $\mathrm{AU}$ & $\mathrm{I}$ & $P^{+}$ & $P^{+}$ & $P^{+}$ & $P^{+}$ & $P^{+}$ & $P^{+}$ & $P^{+}$ & $P^{-}$ & $\mathrm{R}$ & $\mathbf{7}$ & $\mathbf{2} . \mathbf{R}$ \\
$\mathrm{DE}$ & $P^{-}$ & $\mathrm{I}$ & $P^{-}$ & $P^{+}$ & $P^{-}$ & $P^{-}$ & $P^{-}$ & $P^{+}$ & $P^{-}$ & $P^{-}$ & $\mathbf{2}$ & $\mathbf{6 .} \mathbf{R}$ \\
$\mathrm{FI}$ & $\mathrm{I}$ & $P^{+}$ & $\mathrm{I}$ & $P^{+}$ & $P^{+}$ & $P^{-}$ & $P^{+}$ & $P^{+}$ & $P^{-}$ & $\mathrm{R}$ & $\mathbf{5}$ & $\mathbf{3 . ~ R}$ \\
$\mathrm{FR}$ & $P^{-}$ & $P^{-}$ & $P^{-}$ & $\mathrm{I}$ & $P^{-}$ & $P^{-}$ & $P^{-}$ & $P^{+}$ & $P^{-}$ & $P^{-}$ & $\mathbf{1}$ & $\mathbf{7 . ~ R}$ \\
$\mathrm{GB}$ & $P^{-}$ & $P^{+}$ & $P^{-}$ & $P^{+}$ & $\mathrm{I}$ & $P^{-}$ & $P^{-}$ & $P^{+}$ & $P^{-}$ & $P^{-}$ & $\mathbf{3}$ & $\mathbf{5 . ~ R}$ \\
$\mathrm{CA}$ & $P^{+}$ & $P^{+}$ & $P^{+}$ & $P^{+}$ & $P^{+}$ & $\mathrm{I}$ & $P^{+}$ & $P^{+}$ & $P^{-}$ & $\mathrm{R}$ & $\mathbf{7}$ & $\mathbf{2} \mathbf{R}$ \\
$\mathrm{NL}$ & $P^{-}$ & $P^{+}$ & $P^{-}$ & $P^{+}$ & $P^{+}$ & $P^{-}$ & $\mathrm{I}$ & $P^{+}$ & $P^{-}$ & $P^{-}$ & $\mathbf{4}$ & $\mathbf{4 . ~ R}$ \\
$\mathrm{AT}$ & $P^{-}$ & $P^{-}$ & $P^{-}$ & $P^{-}$ & $P^{-}$ & $P^{-}$ & $P^{-}$ & $\mathrm{I}$ & $P^{-}$ & $P^{-}$ & $\mathbf{0}$ & $\mathbf{9} \mathbf{R}$ \\
$\mathrm{CH}$ & $P^{+}$ & $P^{+}$ & $P^{+}$ & $P^{+}$ & $P^{+}$ & $P^{+}$ & $P^{+}$ & $P^{+}$ & $\mathrm{I}$ & $P^{+}$ & $\mathbf{9}$ & $\mathbf{1} \mathbf{R}$ \\
$\mathrm{US}$ & $\mathrm{R}$ & $P^{+}$ & $\mathrm{R}$ & $P^{+}$ & $P^{+}$ & $\mathrm{R}$ & $P^{+}$ & $P^{+}$ & $P^{-}$ & $\mathrm{I}$ & $\mathbf{5}$ & $\mathbf{3 . R}$ \\
\hline & & & & & & & & & & & &
\end{tabular}

oder ob sie indifferent bzw. unvergleichbar sind. Insgesamt gibt es vier mögliche Ausprägungen, die nachfolgend in Tab. 11 definiert werden:

Die Rankingmatrix ergibt sich gemäß der obigen Definitionen (Tab. 11). Demnach sind CA und FI sowie US und GB nicht miteinander vergleichbar (farblich grau hinterlegt). Die Bestimmung des finalen Rankings erfolgt für jede Alternative durch die zeilenweise Addition der Präferenzsituationen, in denen Alternative $a_{\mathrm{i}}$ gegenüber $a_{\mathrm{j}}$ zu präferieren ist. Diese Situationen sind in der Rankingmatrix mit „,P““ gekennzeichnet (Tab. 12).

Die finale Rangordnung wird durch einen bewerteten Graphen wie in Abb. 7 dargestellt. Die Pfeile signalisieren die Dominanz einer Alternative über eine andere. Besteht keine Verbindung zwischen Alternativen, sind diese unvergleichbar, wie es bei AU und US, FI und US sowie US und CA der Fall ist.

\section{Modellanwendung im Immobilienportfoliomanagement}

Die marktbezogenen Modellergebnisse sind im Immobilienportfoliomanagement zu interpretieren und für taktische Anlageentscheidungen zu nutzen. Dabei zielt das Modell nicht auf eine einseitige Investmentstrategie ab. Vielmehr verbindet es auf ei- 


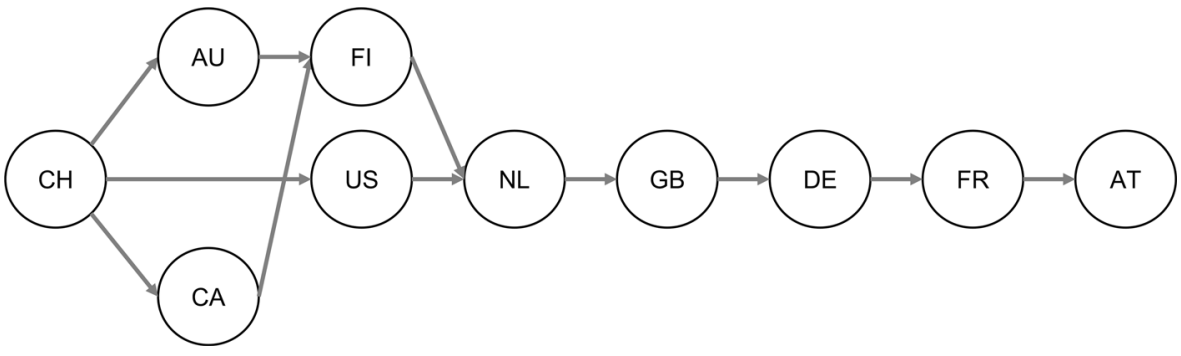

Abb. 7 Finale Rangordnung. (Quelle: Eigene Darstellung)

ner taktischen Zwischenebene die übergeordneten portfoliobezogenen Modelle (z. B. Portfolio Selection) mit der objektbezogenen, fallspezifischen Investitionsrechnung (z. B. Vollständige Finanzpläne). Implikationen im taktischen Bereich können personelle Verstärkungen in priorisierten Zielmärkten sein (Bereich Akquisition, Asset Management) oder eine Absicherungsstrategie in kurz- bis mittelfristig ungünstig bewerteten Zielmärkten (Bereich Risikomanagement, Vertragsmanagement). In diesem Sinne sind die genutzten Bewertungskriterien so zu wählen, dass dieses Modell der taktischen Ebene die strategischen Kriterien (z. B. langfristiges Marktwachstum, Rechtsicherheit) einerseits und die operativen Kriterien (z. B. Verfügbarkeit von Immobilien, Partnern) andererseits nicht überlagert bzw. konterkariert. Im Beispiel erfolgte dies durch Integration grundsätzlicher Parameter (z. B. Rechtsstaatlichkeit) und zyklus- bzw. timing-bezogene Aspekten (z. B. BIP-Wachstum). Im Vergleich zu einem früheren Modelltest führte dies bspw. zu einer Verbesserung der Zielmärkte USA und Kanada, ohne dass eine grundsätzliche Neuordnung stattfand (Metzner und Sawitzky 2015, S. 177). Zusätzliche operative Parameter sind bei der Definition von Kriterien und Vetofunktionen denkbar, das Modell ist dahingehend nicht beschränkt.

Neben dem vorgestellten Szenario einer Zielmarktpriorisierung können ähnliche Modelle für andere immobilienökonomische Standort- und Investitionsentscheidungen entwickelt werden. Die Vorgehensweise ist übertragbar: Demnach müssen je nach Art der Entscheidung (z. B. international, national, überregional, regional, lokal) die Anzahl der Alternativen festgelegt, geeignete Kriterien herangezogen und die Parameter des ELECTRE III-Verfahrens (Schwellenwerte und Gewichte) bestimmt werden.

Auch eine mehrstufige Modellierung ist als Ausbaustufe möglich. Ausgehend von der Zielmarktauswahl könnten auf einer zweiten Stufe Städte bzw. Ballungsräume untersucht werden. Auf dieser Ebene werden spezifische Kriterien benötigt, beispielsweise zur Kaufkraft, Zentralität, Verkehrsanbindung und Branchenstruktur. Auch teilmarktbezogene Kennzahlen wie Marktgröße, Mieten, Renditen und Leerstände spielen eine Rolle (Schilling 2008, S. 256). Je nach Markt müssen die spezifischen Modelle ggf. raumbezogen (landesspezifisch) oder gegenstandbezogen (Nutzungsart, Immobilientyp) angepasst werden.

Eine weitere Stufe der Modellanwendung ist auf der Objekt- bzw. Investitionsebene denkbar. Diese würde eine Priorisierung von konkreten Investitionsalternativen anhand qualitativer und quantitativer Kriterien wie Objektrendite, Miete, Leerstand, 
Neben- und Instandhaltungskosten, Bausubstanz, Architektur, Gebäudeausstattung, Flexibilität usw. beinhalten.

\section{Ergebnisse und Schlussfolgerungen}

In der vorliegenden Studie wurde ELECTRE III als alternatives Modell zur Lösung klassischer Bewertungs- und Entscheidungsprobleme im taktischen Immobilienportfoliomanagement vorgestellt. Das Entscheidungsproblem „Priorisierung von Zielmärkten" diente der beispielhaften Darstellung der Verfahrensschritte, Parameter und Ergebnisse. Zur Begrenzung der Modellkomplexität und zur besseren Übersichtlichkeit basierten die verwendeten Entscheidungsalternativen und Entscheidungskriterien auf vorläufigen Annahmen. Im praktischen Einsatz können weitere Alternativen und Kriterien hinzugefügt werden. Grundsätzlich bietet das gut programmierbare Verfahren die Möglichkeit, auch eine große Anzahl von Alternativen und Kriterien zu verarbeiten. Eine Beschränkung ist durch die Anzahl unvergleichbarer Alternativen gegeben, die mit zunehmender Komplexität zu erwarten sind.

Anders als bei den bisher in der Immobilienbranche - sowohl in der Praxis (Subroweit 2008; Piasecki und Embacher 2014; Beyerle et al. 2015) als auch in wissenschaftlichen Publikationen (Bone-Winkel 1994, S. $199 \mathrm{ff}$, Urschel 2010, S. $276 \mathrm{ff}$ ) - noch häufig zu findenden Scorings (Metzner 2013, S. 372) kann auf manuelle Bewertungen in der Modellanwendung verzichtet werden (unter Akzeptanz einer ggf. aufwändigerer Modellentwicklung), womit in den entsprechenden Entscheidungssystemen trotz einer sehr differenzierteren und exakten Methodik eine hinreichende Effizienz und Objektivität gegeben ist. Einzelne subjektive Festlegungen (bspw. Schwellenwerte) werden transparent dargestellt. Der parallele Bezug zu quantitativen und qualitativen (jedoch quantifizierbaren) Kriterien ist in ELECTRE III unproblematisch, so dass zahlreiche Fragestellungen aus dem Bereich Bewertung, Auswahl und Priorisierung unterstützt werden können.

Neben der strategischen Auswahl von Zielmärkten kann das Modell somit auf eine Vielzahl immobilienökonomischer Entscheidungssituationen adaptiert werden. Naheliegend ist die Weiterentwicklung bereits vorhandener qualitativer Analysemethoden, etwa von gewichtungsbasierten Scorings oder Ratings. Vor allem bei kapitalintensiven Investitions- und Desinvestitionsentscheidungen lohnt sich der Einsatz komplexer immobilienökonomischer Modelle wie ELECTRE III. Zu beachten sind selbstverständlich der Entwicklungsaufwand, aber auch die Akzeptanz von Outranking bzw. ELECTRE im Unternehmen. Die damit generierte unvollständige Rangordnung mit fallweise unvergleichbaren Alternativen kann beim Entscheider Fragen zur ,tatsächlichen“ Rangfolge aufwerfen. Die Vorteile von ELECTRE-Verfahren liegen jedoch gerade in dieser realitätsnahen Betrachtungsweise von Alternativen und der Erweiterung des Präferenzbegriffes. Sehr unterschiedliche Alternativen lassen sich auch tatsächlich nicht immer direkt vergleichen, da sich zahlreiche Kriterien nicht immer kompensieren und zusammenfassen lassen. Dies gilt insbesondere für kritische Kriterien wie Markttransparenz und Rechtsystem, die bisher vorgelagert (gesondert) zum eigentlichen Entscheidungsmodell zu betrachten sind. 
Die weitere wissenschaftliche bzw. individuell im Unternehmen durchzuführende Entwicklungsarbeit wird sich vorrangig auf die Bestimmung und Optimierung der Modellparameter konzentrieren (Auswahl, Gewichtung, Schwellenwert, ...). Auch die generelle Methodik von ELECTRE ist Gegenstand der weiteren Forschung (Figueira et al. 2013, S. 71 ff). Generell bietet die Übertragung von Modellen und Verfahren der Entscheidungsunterstützung auf Fragestellungen der Immobilienökonomie noch ein breites Forschungspotenzial. Die vorgestellte Modellierung auf Basis von ELECTRE III steht an dieser Stelle beispielhaft für die vielfältigen Möglichkeiten in diesem Umfeld.

Interessenkonflikt S. Metzner und M. Sawitzky geben an, dass kein Interessenkonflikt besteht.

Open Access Dieser Artikel wird unter der Creative Commons Namensnennung 4.0 International Lizenz (http://creativecommons.org/licenses/by/4.0/deed.de) veröffentlicht, welche die Nutzung, Vervielfältigung, Bearbeitung, Verbreitung und Wiedergabe in jeglichem Medium und Format erlaubt, sofern Sie den/die ursprünglichen Autor(en) und die Quelle ordnungsgemäß nennen, einen Link zur Creative Commons Lizenz beifügen und angeben, ob Änderungen vorgenommen wurden.

\section{Literatur}

Adair A, Berry J, McGreal S, Sýkora L, Parsa A, Redding B (1999) Globalization of real estate markets in Central Europe. Eur Plan Stud 7(3):295-305

Bächle M (1996) Qualitätsmanagement der Softwareentwicklung. Deutscher Universitäts-Verlag, Wiesbaden

Benayoun R, Roy B, Sussman B (1966) ELECTRE: Une m'ethode pour guider le choix en pr'esence de points de vue multiples. Note de travail 49. SEMA-METRA International, Direction Scientifique, Paris

Beyerle T, Müller L, Slupik A, Zweigner F (2015) Catella Research - Wohnungsmarktranking Deutschland 2015: Die regionalen Wohnungsmärkte unter dem Mikroskop. www.catella.com/PageFiles/33110/ Catella\%20Research\%20Wohnungsmarktranking\%202015.pdf. Zugegriffen: 15. Juni 2016

Bone-Winkel S (1994) Das strategische Management von offenen Immobilienfonds unter besonderer Berücksichtigung der Projektentwicklung von Gewerbeimmobilien. Schriften zur Immobilienökonomie, Bd. 1. Müller, Köln

Botti L, Peypoch N (2013) Multi-criteria ELECTRE method and destination competitiveness. Tour Manag Perspect 6:108-113

Buchanan J, Vanderpooten D (2007) Ranking projects for an electricity utility using ELECTRE III. Int Trans Oper Res 14(4):309-323

Chen J, Hobbs P (2003) Global Real Estate Risk Index. J Portfolio Manag 29(5):66-75

Chin W, Dent P, Roberts C (2006) An exploratory analysis of barriers to investment and market maturity in Southeast Asian cities. J Real Estate Portf Manag 12:49-57

Damaskos X, Kalfakakou G (2005) Application of ELECTRE III and DEA methods in the BPR of a bank branch network. Yugosl J Oper Res 15(2):259-276

D’Arcy É, Keogh G (1998) Territorial competition and property market process: an exploratory analysis. Urban Stud 35(8):1215-1230

Falkenbach H (2009) Market selection for international real estate investments. Int J Strateg Prop Manag 13(4):299-308

Falkenbach H (2010) Motivation and market selection of international investors in Finnish property market. Nord J Surv Real Estate Res 7(1):15-29

Figueira JR, Mousseau V, Roy B (2005) ELECTRE methods. In: Figueira JR, Greco S, Ehrgott M (Hrsg) Multiple criteria decision analysis - state of the art surveys. Springer, Heidelberg, S 133-162

Figueira JR, Greco S, Roy B, Słowiński R (2013) An overview of ELECTRE methods and their recent extensions. J Multicriteria Decis Anal 20(1-2):61-85. doi:10.1002/mcda.1482

Gäfgen G (1966) Theorie der wirtschaftlichen Entscheidung, 3. Aufl. Mohr Siebeck, Tübingen

Geldermann J (1999) Entwicklung eines multikriteriellen Entscheidungsunterstützungssystems zur integrierten Technikbewertung. VDI-Verlag, Düsseldorf 
Geurts TG, Jaffe AJ (1996) Risk and real estate investment - an international perspective. J Real Estate Res 11(2):117-130

Giannoulis C, Ishizaka A (2010) A web-based decision support system with ELECTRE III for a personalised ranking of British Universities. Decis Support Syst 48(3):488-497

Hoskins N, Higgins D, Cardew R (2004) Macroeconomic variables and real estate returns: an international comparison. Appraisal J 72(2):163-170

Huang W, Chen C (2005) Using the ELECTRE II method to apply and analyze the differentiation theory. Proc East Asia Soc Transp Stud 5:2237-2249

Ishizaka A, Nemery P (2013) Multi-criteria decision analysis: methods and software. Wiley, Chichester

Keogh G, D'Arcy É (1994) Market maturity and property market behaviour: a European comparison of mature and emergent markets. J Prop Res 11(3):215-235. doi:10.1080/09599919408724118

Keogh G, D’Arcy É (1999) Property market efficiency: an institutional economics perspective. Urban Stud 36(13):2401-2414

Knack S, Keefer P (1995) Institutions and economic performance: cross-country tests using alternative institutional measures. Econ Polit 7(3):207-227

Köpplinger H, Wolfrum B (1986) Risiken im Auslandsgeschäft. Eine theoretische und empirische Untersuchung des politischen Investitionsklimas Bd. 1. Pauli-Balleis, Zirndorf

Lee S (2001) The risk of investing in the real estate markets of the Asian region. Working Paper. University of Reading, Reading

Lee S (2005) Gauging the investment potential of international real estate markets. A paper presented at the Annual European Real Estate Society Meeting (ERES) Dublin. http://centaur.reading.ac.uk/20959/1/ 1905.pdf. Zugegriffen: 28. August 2014

Lieser K (2012) The attractiveness of countries for international real estate investments - the global real estate investment attractiveness index. Immobilien Manager Verlag, Köln

Lifka S (2009) Entscheidungsanalysen in der Immobilienwirtschaft - Wirtschaft und Raum. Utz, München

Lim LC, McGreal S, Webb JR (2006) Perception of real estate investment opportunities in Central/South America and Africa. J Real Estate Portfolio Manag 12(3):261-276

Lynn DJ (2007) The tectonic forces of global real estate: implications for global investment and portfolio managers. J Real Estate Portfolio Manag 13(1):87-92

Maier KM (2007) Risikomanagement im Immobilien- und Finanzwesen - Ein Leitfaden für Theorie und Praxis. Fritz Knapp, Frankfurt a. M.

Mauro P (1995) Corruption and Growth. Q J Econ 110(3):681-712

Metzner S (2013) Immobilienökonomische Methoden zur Entscheidungsunterstützung und Planung - Selektion und Integration im Rahmen der wertorientierten Steuerung von Immobilienportfolios institutioneller Anleger. Monsenstein und Vannerdat, Münster

Metzner S, Sawitzky M (2015) Moderne Instrumente des Immobiliencontrollings IV - ELECTRE-Modell zur Priorisierung von Immobilieninvestments. Books on Demand, Norderstedt

Nastansky A (2012) Wechselbeziehnungen zwischen Immobilienmarkt und Makroökonomie. In: Rottke N, Voigtländer M (Hrsg) Ökonomie. Immobilienwirtschaftslehre, Bd. II. Immobilien Manager Verlag, Köln, S 163-214

Nielsen L (2011) Classifications of countries based on their level of development: How it is done and how it could be done. IMF Working Paper. International Monetary Fund, Washington DC

Piasecki H, Embacher F (2014) Makro-Scoring Studentenwohnen - Neues Makro-Scoring für die Assetklasse Studentenwohnen. http://www.bulwiengesa.de/sites/default/files/makro-scoring_ studentenwohnen_140807.pdf. Zugegriffen: 1. August 2015

Reichsthaler T, Siegel G (2009) Kreditrisikosteuerung und Rating: vom Immobilienmarkt zum Objekt. In: Junis K, Piazolo D (Hrsg) Praxishandbuch Immobilienmarktrisiken. Immobilien Manager Verlag, Köln, S 473-498

Rogers M (2000) Using Electre III to aid the choice of housing construction process within structural engineering. Constr Manag Econ 18(3):333-342

Roussat, N, Dujet C, Méhu J (2009) Choosing a sustainable demolition waste management strategy using multicriteria decision analysis. Waste Management 29(1):12-20

Roy B (1980) Selektieren, Sortieren und Ordnen mit Hilfe von Prävalenzrelationen - Neue Ansätze auf dem Gebiet der Entscheidungshilfe für Multikriteria-Probleme. Zeitschrift Für Betriebswirtschaftliche Forsch 32:465-497

Roy B (1991) The outranking approach and the foundations of ELECTRE methods. Theory Decis 31(1):49-73

Roy B, Présent M, Silhol D (1986) A programming method for determining which Paris metro stations should be renovated. Eur J Oper Res 24:318-334 
Schilling T (2008) Research für die Globale Asset Allocation bei offenen Immobilien- und ImmobilienAktienfonds. In: Junis K, Piazolo D (Hrsg) Praxishandbuch Immobilien-Research. Immobilien Manager Verlag, Köln, S 243-264

Subroweit S (2008) Der Deka Immobilien Real Estate Country Score (DIRECS) - Immobilien Research Indikatoren. https://www.deka-s-propertyfund-no1.de/content/e35/e1290/e1291/e2498/e1309/

MultiFile1310/DIRECS2008_beurteilung_immob_ger.pdf. Zugegriffen: 21. August 2014

Subroweit S (2009) Risikoscoring von Gewerbeimmobilienmärkten. In: Junius K, Piazolo D (Hrsg) Praxishandbuch Immobilienmarktrisiken. Immobilien Manager Verlag, Köln, S 279-300

Transparency International (2013) Corruption perceptions index 2014. http://cpi.transparency.org/cpi2013/ in_detail/abgerufen. Zugegriffen: 15. Juli 2015

Trotz R (2004) Immobilien-Markt- und Objektrating: Ein praxiserprobtes System für die Immobilienanalyse. Rudolf Müller, Köln

United Nations (2015) Member States - growth in United Nations membership, 1945-present. http://www. un.org/en/sections/member-states/growth-united-nations-membership-1945-present/index.html. Zugegriffen: 10. Juli 2015

Urschel O (2010) Risikomanagement in der Immobilienwirtschaft - Ein Beitrag zur Verbesserung der Risikoanalyse- und -bewertung. KIT Scientific Publishing, Karlsruhe

Webb JR (1984) Real estate investment acquisition rules for life insurance companies and pension funds: a survey. J Am Real Estate Urban Econ Assoc 12(4):495-520

Wellner K (2003) Entwicklung eines Immobilien-Portfolio-Management-Systems: Zur Optimierung von Rendite-Risiko-Profilen diversifizierter Immobilien-Portfolios. Institut für Immobilienmanagement, Universität Leipzig, Leipzig

Worzala E (1994) Overseas property investments: How Are they perceived by the institutional investor? J Prop Valuat Invest 12(3):31-47

Worzala E, Bernasek A (1996) European economic integration and commercial RE markets: an analysis in market determinants. J Real Estate Res 11(2):159-181

Zimmermann H-J, Gutsche L (1991) Multi-criteria-Analyse - Einführung in die Theorie der Entscheidungen bei Mehrfachzielsetzungen. Springer, Berlin 